\title{
Performance Comparison of PIN and APD based FSO Satellite Systems for various Pulse Modulation Schemes in Atmospheric Turbulence
}

\author{
Pooja Gopal $^{1}$, V. K. Jain ${ }^{2}$, Subrat Kar ${ }^{2}$ \\ ${ }^{1}$ Bharti School of Telecom Tech. and Mgmt., IIT Delhi, New Delhi, India \\ ${ }^{2}$ Electrical Engineering Dept., IIT Delhi, New Delhi, India \\ Email: pooja.gopal@dbst.iitd.ac.in
}

Received June, 2013

\begin{abstract}
In this paper, the performance of various Pulse Position Modulation (PPM) schemes has been analysed for PIN and APD receivers in the presence of atmospheric turbulence. It is observed that the performance of the APD receiver is always better than that of the PIN receiver as expected. Among the various modulation schemes, the performance of Differential Amplitude PPM (DAPPM) scheme with more number of amplitude levels is better than that of the other schemes for the same single level peak amplitude. Further, the optimum gain of APD receiver does not change substantially for different modulation schemes and turbulent conditions.
\end{abstract}

Keywords: Free Space Optics; Pulse Modulation; Ground-to-satellite Communications

\section{Introduction}

Terrestrial Free Space Optical (FSO) systems such as optical fibre backup links, cellular communication backhaul links, multi-campus links, etc. have been performing well right from their emergence. Inter-satellite FSO links have already been established, while the FSO link between a ground station and an orbiting satellite is being extensively reported $[1,2]$.

The major advantages offered by this technology over the conventional Radio Frequency (RF) satellite systems are the following: (i) the size and weight of the payload are critical parameters in any satellite system. These parameters in FSO systems are one third less than that of corresponding RF systems [3], (ii) the beam divergence angle in RF systems is large, which results in large footprints. This limits the number of satellites using the same spectrum and also poses a threat to the security of such systems. In contrast to this, the extremely small beam widths and divergence angles of lasers offer links which are basically immune to interference and offer high security, and (iii) there are as yet no restrictions on the bandwidth used in FSO systems. Further, they offer very high data rates which are virtually unconstrained by the carrier frequencies.

Pulse Position Modulation (PPM) is very popular in long distance optical communication systems because of its high power efficiency. Also, due to its high Peak to
Average Power Ratio (PAPR), it is resilient to the effects of noise. The bandwidth requirement which increases with the order of the PPM, is directly proportional to the power efficiency i.e., higher order PPM schemes are more power efficient and resilient to noise. Ground-tosatellite optical communications have to undergo the ill effects of atmospheric turbulence, the amount of which is proportional to the strength of turbulence. The pulse broadening limits the rate at which data can be sent through the turbulent channel. Hence, PPM may not be an ideal choice of modulation scheme in all atmospheric conditions because of its high bandwidth requirement. Several variants of PPM scheme have been in use, which offer a trade-off between the power efficiency and bandwidth efficiency. In this paper, the comparison of the Bit Error Rate $(B E R)$ performance of the various PPM schemes is analysed.

This paper is organized as follows. In Section 2, a brief description of the PPM schemes is given. The Section 3 contains the system model used for subsequent analysis. The methodology for system performance evaluation and the numerical results in graphical form are presented in Section 4. The conclusions of the study are given in Section 5.

\section{Modulation Schemes}

In Differential Pulse Position Modulation (DPPM), all the empty slots following the pulse in PPM are removed. 
This reduces the average symbol length implying improved bandwidth efficiency. Also, there is an inherent symbol synchronization capability as every symbol ends with a pulse. Like PPM and unlike On-Off Keying (OOK), DPPM does not require an adaptive threshold at the receiver.

Differential Amplitude Pulse Position Modulation (DAPPM) is a combination of DPPM and Pulse Amplitude Modulation (PAM). The average number of empty slots following a pulse in DPPM can be reduced by increasing the number of amplitude levels $A$. This in turn increases the bandwidth efficiency. But, it adds the requirement of having an adaptive threshold, due to the presence of multi-amplitudes. A well designed DAPPM system would require less bandwidth in comparison to OOK, PPM and DPPM systems [2]. It has inherent symbol synchronization capability like DPPM.

\section{System Model}

The three most reported models for irradiance fluctuations in a turbulent channel are: log-normal, gammagamma and negative exponential. Their respective ranges of validity are in the weak, weak-to-strong and saturation regimes. In the region of weak fluctuations, the statistics of the irradiance fluctuations have been experimentally found to obey the log-normal distribution [4]. The probability density function of log-normal distribution is given by

$$
p(I)=\frac{1}{\sqrt{2 \pi \sigma_{I}^{2}}} \frac{1}{I} \exp \left\{-\frac{\left(\ln \left(I / I_{0}\right)-E[I]\right)^{2}}{2 \sigma_{I}^{2}}\right\}, I \geq 0
$$

and the scintillation index $\sigma_{I}^{2}$ is given by the expression

$$
\sigma_{I}^{2}=\frac{E\left[I^{2}\right]}{E^{2}[I]}-1
$$

where $I$ is the received field intensity in presence of turbulence and $I_{0}$ the received field intensity without the effect of turbulence, $\sigma_{I}^{2}$ the log-intensity variance and $E[I]$ the mean of log-intensity variance. For the case of strong turbulence, the probability density function is given by the negative exponential distribution

$$
p(I)=\frac{1}{I_{0}} \exp \left\{-\frac{I}{I_{0}}\right\}
$$

The Avalanche Photo Diode (APD) is generally used in long distance optical communications because of the low received power levels. An APD performs better than a PIN diode receiver, when the received power levels are low. A high avalanche gain requires a high reverse bias voltage. The higher gain doesn't imply a better signal to noise ratio (SNR) since the performance degrades beyond a certain gain as the effect of noise becomes dominant. Hence the optimum gain of APD for the particular sys- tem has to be used. A comparative study of APD receiver vis-à-vis PIN receiver is made in the following section.

\section{Methodology for System Performance Evaluation}

The number of photons received at the detector, $N_{s}$ would be a log-normal distributed random variable (in the case of weak turbulence) or a negative exponentially distributed random variable (in the case of strong turbulence). The conditional Bit Error Rate (BER) is then given by

$$
P_{b / i}=\frac{1}{2} \operatorname{erfc}\left[\frac{Q\left(N_{s}\right)}{\sqrt{2}}\right]
$$

where $Q\left(N_{s}\right)$ is the $Q$-parameter. The unconditional $B E R$ is then given by

$$
B E R=\int_{0}^{\infty} P_{b} p\left(N_{s}\right) d N_{s}
$$

where $p\left(N_{s}\right)$ is the probability density function of $N_{s}$. Since, $N_{s}$ is proportional to the received irradiance $I$, $p\left(N_{s}\right)$ can be determined from eqn. (1) or (3) depending on the level of turbulence. After simplification using the Gauss-Hermite approximation [6], the corresponding $B E R$ expressions are obtained. The BER expressions for the different modulation schemes are derived by taking into consideration the respective bandwidth and power requirements. The Symbol Error Rate (SER) expressions are obtained from the respective $B E R$ expressions.

The SER expressions for PIN and APD receivers when different modulation schemes are used are obtained from eqs. (1)-(5). The modulation schemes considered are 64-PPM, 64-DPPM, 64-DAPPM ( $A=2, L=32 ; A=4, L=16$ and $A=8, L=8)$. The numerical results computed from these expressions are shown in Figures 1-4. Figures 1(a)-(b) and Figure 2(a) give the graphs of $S E R$ vs. $N_{s}$ (in dB) for the PIN receiver for $\sigma_{s c}^{2}=0$ (no turbulence), 0.5 (low turbulence) and 1 (high turbulence), respectively. The corresponding graphs for the APD receiver are given in Figures 2(b), Fiugres 3(a) and (b), respectively.

We observe from Figure 1(a) that the performance of DPPM is better than that of PPM. Further, the performance of DAPPM is better than that of DPPM and PPM. The performance of DAPPM becomes still better if the number of levels is increased from 2 to 8 . This trend remains the same irrespective of the turbulence level. In case of APD receiver, the comparative performance of different modulation schemes is similar to that of the PIN case. But, the required $S N R$ to obtain a particular $S E R$ is much less than that of the PIN receiver.

In Figures 4(a) and (b), the variations of $S E R$ vs. APD gain are given for low turbulence and high turbu- 
lence cases, respectively. It is observed that there is not much difference in the optimum gain for different modulation schemes. The optimum gain for different turbu-

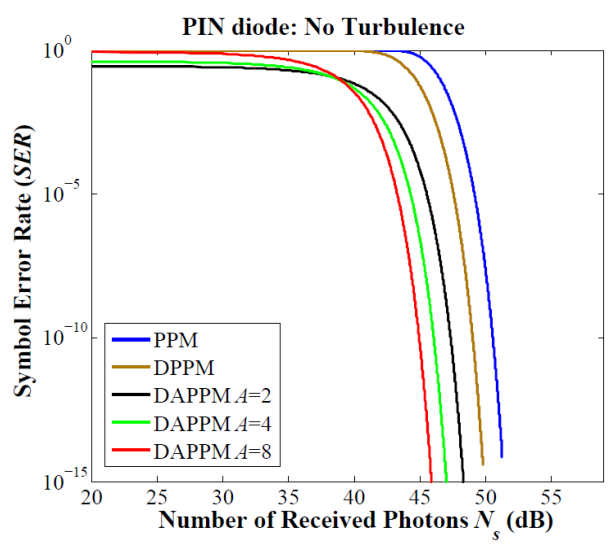

(a) lence conditions is almost same. But, we see that the degradation in performance with increase in gain, beyond the optimum gain is more in the case of high turbulence.

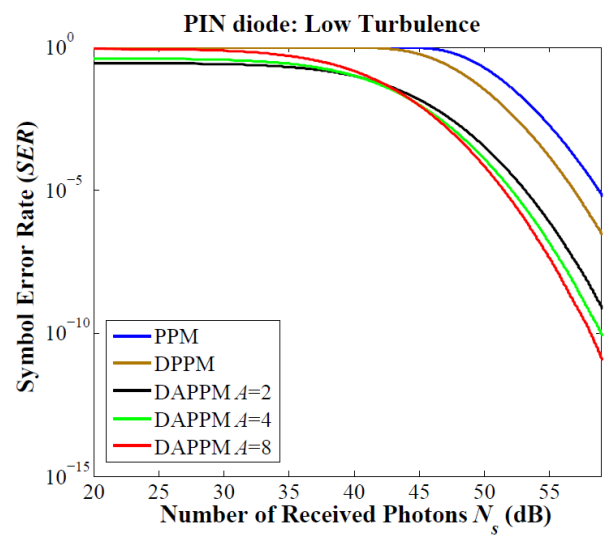

(b)

Figure 1. (a) $S E R$ vs. $N_{s}$ in PIN receiver for different modulation schemes without turbulence; (b) $S E R$ vs. $N_{s}$ in PIN receiver for different modulation schemes in low turbulence.

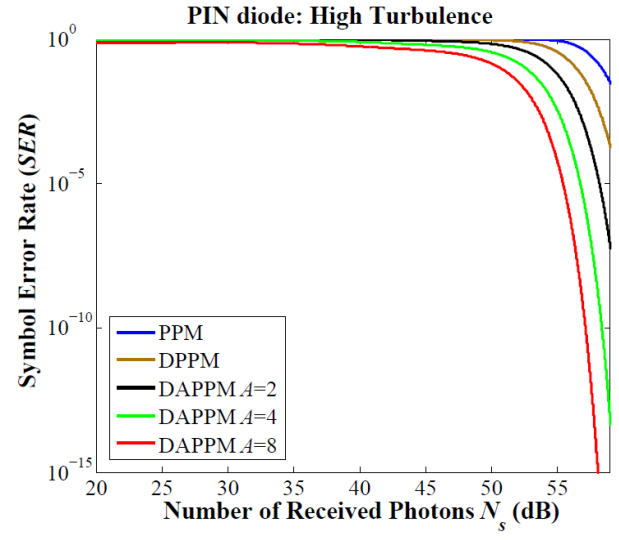

(a)

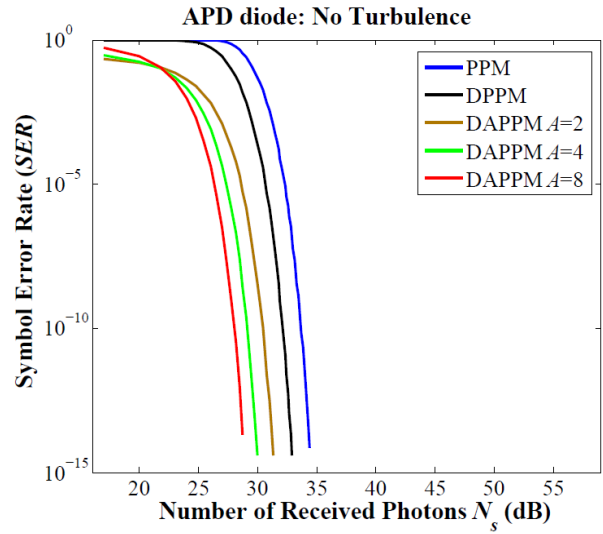

(b)

Figure 2. (a) $S E R$ vs. $N_{s}$ in PIN receiver for different modulation schemes in high turbulence; (b) $S E R$ vs. $N_{s}$ in APD receiver for different modulation schemes without turbulence.

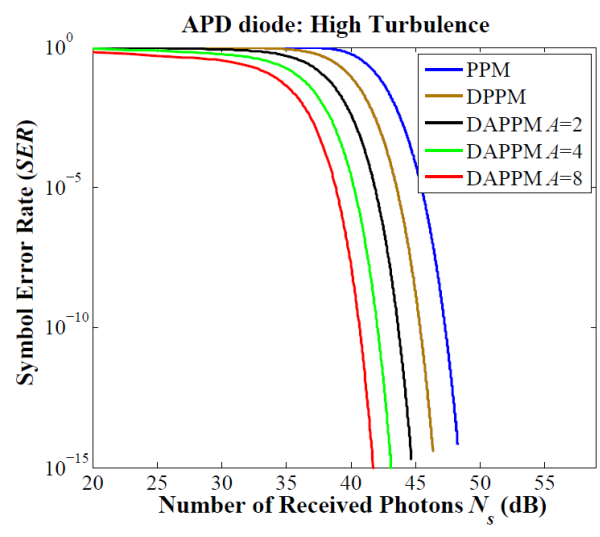

(a)

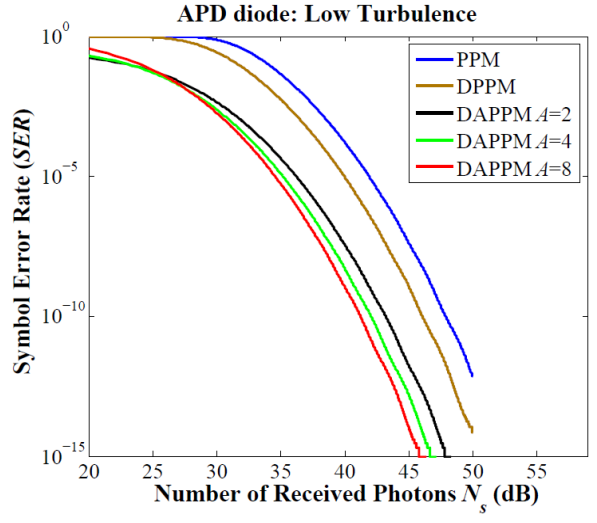

(b)

Figure 3. (a) $S E R$ vs. $N_{s}$ in APD receiver for different modulation schemes in low turbulence; (b) $S E R$ vs. $N_{s}$ in APD receiver for different modulation schemes in high turbulence. 


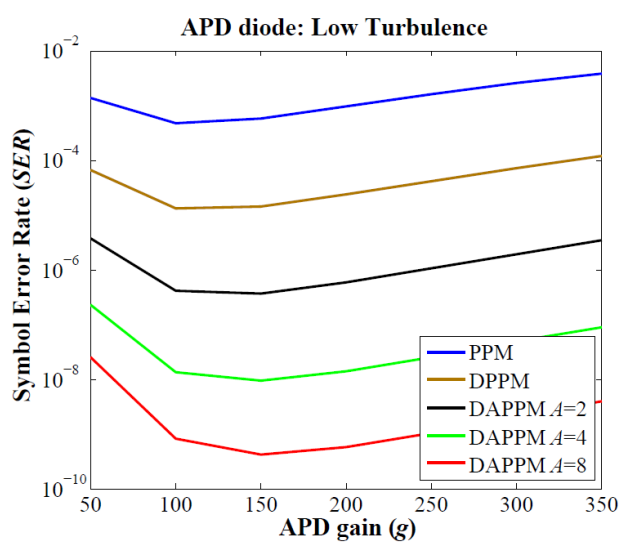

(a)

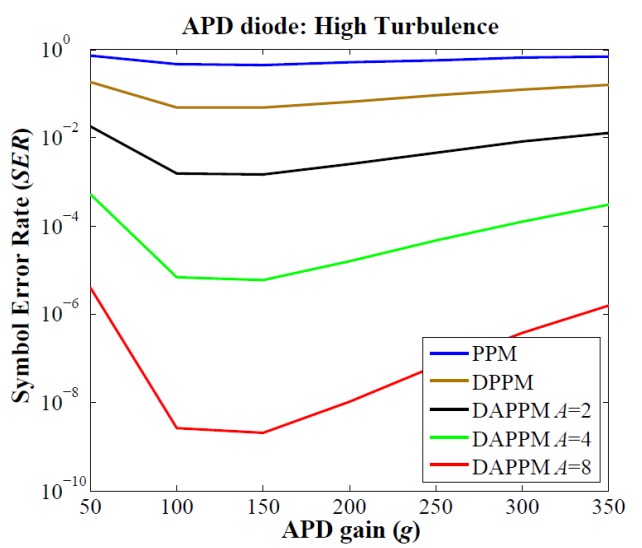

(b)

Figure 4. (a) $S E R$ vs. $g$ in APD receiver for different modulation schemes in low turbulence; (b) $S E R$ vs. $g$ in APD receiver for different modulation schemes in high turbulence.

Table 1. Parameter values in numerical computation.

\begin{tabular}{lc}
\hline \multicolumn{1}{c}{ Parameter } & Value \\
\hline Bit rate $(1 / T)$ & $1 \mathrm{Gbps}$ \\
Receiver temperature $\left(T_{0}\right)$ & $300 \mathrm{~K}$ \\
Average APD gain $(g)$ & 150 \\
Load resistance $\left(R_{L}\right)$ & $100 \Omega$ \\
APD noise figure & 6.756 \\
Ionization factor & 0.02 \\
Order of PPM $(M)$ & 64 \\
\hline
\end{tabular}

The numerical values of the parameters used in the computation are given in Table 1.

\section{Conclusions}

The performance of APD receiver is much better than that of corresponding PIN receiver. This is because of the gain factor and also the low received power levels. Hence for satellite communications which involve long distances implying less received power, APD receivers are more suitable. Also, there is no substantial change in the optimum gain for different modulation schemes and atmospheric conditions. The disadvantage is the requirement of high bias voltages for more gains, which will add to the payload.

The better performance of the DAPPM scheme as compared to PPM and DPPM schemes can be attributed to the fact that more number of levels reduce the effective symbol length, which in turn reduces the bit duration and hence inter symbol interference is caused due to turbulence. This also explains why DAPPM schemes with more number of levels perform better.

The degradation of performance with increase in gain beyond an optimum value is due to the fact that the noise is amplified along with the signal and beyond a certain point, the effect of amplified noise is more than that of the signal. Similarly, the degradation is more in the case of strong turbulence because, turbulence causes random variation of received signal which is amplified similar to the noise.

We can conclude that because of the time varying nature of the atmospheric channel, the performance of the link depends on the turbulence conditions. Hence by adaptively changing the modulation, a more robust system performance can be expected.

\section{REFERENCES}

[1] H. Kaushal, V. K. Jain and S. Kar, "Performance Improvement with Coding of Free Space Optical Ground to Satellite Link in Atmospheric Turbulence Environment," AIP Conference Proceedings, Kerala, 2011.

[2] P. Gopal, V. K. Jain and S. Kar, "Performance Analysis of Ground to Satellite FSO System with DAPPM Scheme in Weak Atmospheric Turbulence,” International Conference on Fibre Optics and Photonics, Chennai, India, WPo. 43, December 2012.

[3] L. C. Andrews and R. L. Phillips, Laser Beam Propagation Through Random Media, $2^{\text {nd }}$ Edition, SPIE Press, Washington, 2005

[4] R. L. Phillips and L. C. Andrews, "Universal Statistical Model for Irradiance Fluctuations in a Turbulent Medium," Journal of Optical Society of America, Vol. 72, No. 7, 1982, pp. 864-870. doi:10.1364/JOSA.72.000864

[5] G. P. Agrawal: Fiber Optic Communication Systems, Wiley, India, 2002. doi:10.1002/0471221147

[6] K. Kiasaleh, "Performance of APD-based, PPM Free Space Optical Communication Systems in Atmospheric Turbulence," IEEE Transactions on Communications, Vol. 53, No. 9, 2005, pp. 1455-1461. doi:10.1109/TCOMM.2005.855009 